\title{
INTRA-OCULAR FILARIASIS IN AUSTRALIA
}

BY

\author{
N. KERKENEZOV \\ Lismore, N.S.W., Australia
}

THE first recorded case of an intra-ocular filaria was published by Mercier (1771). Cases have since appeared sporadically, but unfortunately most have not been supported by a detailed description of the nematode, as this has often evaded capture.

A complete summary of the relevant literature is given by Duke-Elder (1940). The clinical records being so few, the following case history is of interest.

\section{Case Report}

A man aged 54 years was first seen on June 7, 1960, complaining that his left eye had been irritable ("a foreign body sensation"), watery, red, and blurred for the previous 7 days. At times the visual acuity was normal, but at others "it was completely blurred". The past 24 hours had seen a marked increase in symptoms and he now had constant ocular pain associated with a bilateral frontal headache.

Examination.-The visual acuity in the right eye was 6/24 and in the left "perception of light".

The patient was obviously in considerable distress, the left eye being closed because of a blepharospasm. There was an inequality of the pupils caused by a left miosis. To the naked eye, the lower half of the cornea appeared somewhat hazy and the slit lamp showed this to be due not only to oedema of the substantia propria but also to numerous fine epithelial bullae. The endothelium was more prominent than normal and scattered over its inner surface were numerous fine ill-defined deposits. Cells were present in the anterior chamber, but these were not as numerous as one would have expected in such an irritable eye. The upper half of the cornea was normal.

Close examination of the iris revealed laterally two fine filamentary objects (Fig. 1).

FIg. 1.-Examination of iris at time of first consultation revealed two fine filamentary foreign bodies. The impression was gained that these formed part of a whole as indicated by the dotted lines (representing that portion of the filaria which is behind the iris). The filaria had perforated the iris at three points and appeared completely immobile.

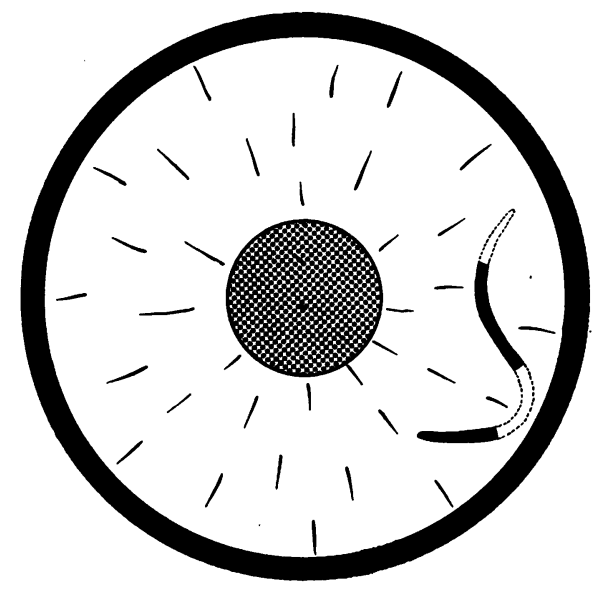

* Received for publication December 20, 1961. 


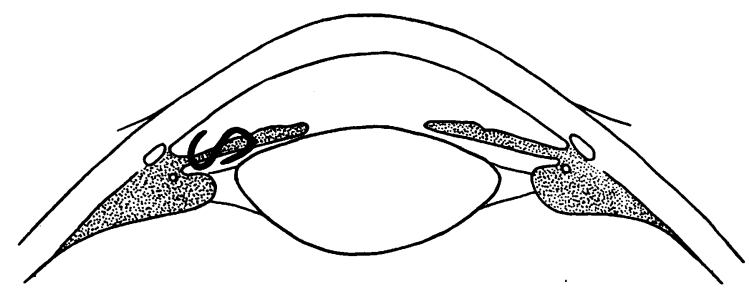

FIG. 2.-Diagrammatic vertical section of anterior portion of globe, showing filaria in relation to iris at time of first consultation.

These gave the impression that they were but part of one thin elongated foreign body which had threaded itself through the iris as indicated in Fig. 2.

Under local mydriasis the lens appeared normal; there was a complete absence of any posterior synechiae and the fundus showed no apparent pathology, although the examination of the latter was rather difficult.

The intra-ocular pressure was normal in each eye ( $25 \mathrm{~mm}$. Hg Schiötz).

The right eye was normal with no lens opacities. The fundus was normal apart from a slight pigmentary macular degeneration.

Treatment.-The patient was admitted to hospital and the case treated as one of iridocyclitis secondary to an ?? intra-ocular filaria. Some 12 hours later the diagnosis was established rather dramatically, for the thread-like object had released itself from the iris and was actively motile within the anterior chamber. With the patient erect, the filaria would at times lie coiled and immobile in the lower reaches of the anterior chamber where its presence was very difficult to establish, but at others, it would swim rapidly throughout the aqueous motivated by rapid coiling and uncoiling motions, striking at times either the posterior surface of the cornea or the anterior surface of the iris. After some seconds of activity, during which the patient suffered acute exacerbation of pain, the filaria would sink to the bottom of the anterior chamber again.

With the patient recumbent, the filaria, after a bout of activity, would fall either on to the anterior lens capsule or on to the iris, according to its position when motion ceased (Fig. 3). It made no attempt to pass between the iris and the anterior lens capsule.

FIG. 3.-Filaria lying within the anterior chamber in a semi-coiled position. At this stage it was freely motile.

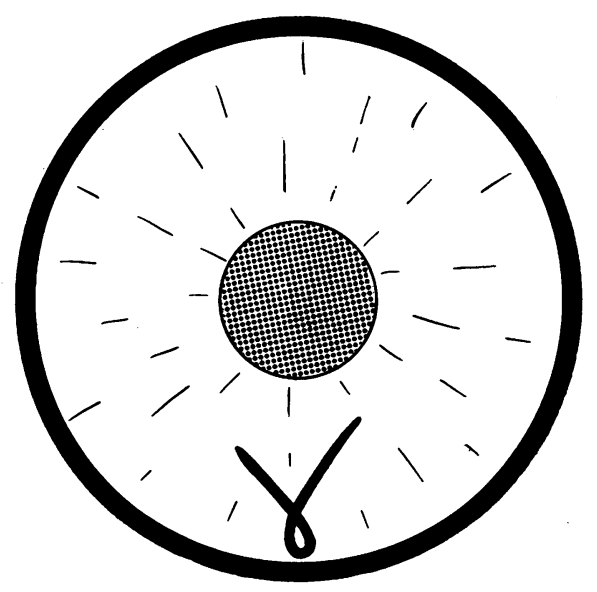

Pilocarpine did not appear to stimulate the filaria to greater activity. rate.

The patient's general condition was normal with normal body temperature and pulse 
Previous History.-Both tobacco and alcohol were indulged in gross excess.

The patient joined the Army in 1941 and served in the Middle East for some 2 years. While in Syria in 1942 he developed pleurisy and bronchitis (hospital for 3 months), and after a recurrence in 1943 he was repatriated and discharged as class B.

On his return to Australia, he worked in both a timber mill and a banana plantation within the Murwillumbah area of New South Wales, and did not leave this area except on the following three occasions:

(1) A visit for one week to Sydney, some 3 weeks before his present illness.

(2) One day in Brisbane, 2 weeks before the ocular lesion developed.

(3) 3 weeks in Brisbane some 6 or 7 years ago.

The relevant latitudes of these areas are shown in Fig. 4.

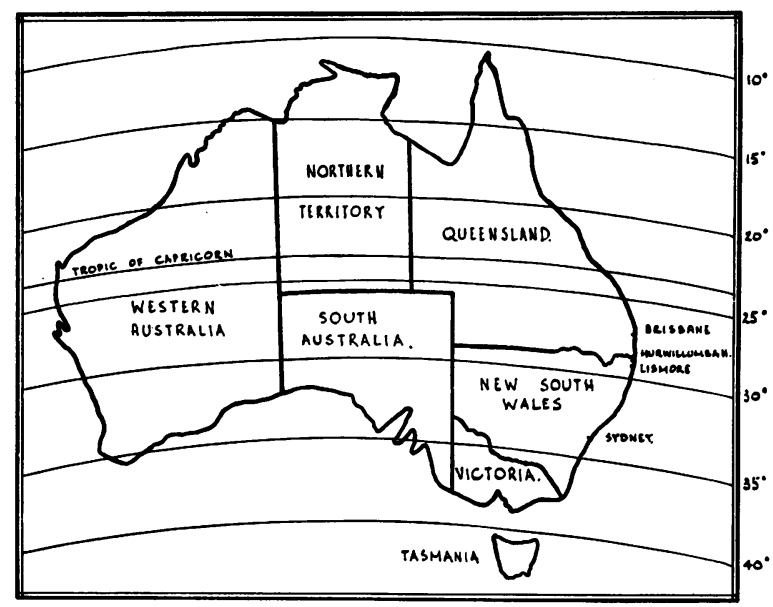

Fig. 4.-Map of Australia, showing the latitudes in relation to the areas (Lismore-Murwillumbah) in which the filariasis apparently orginated.

Pathological Investigations. - On admission a full blood investigation was undertaken and the report was as follows:

Erythrocyte sedimentation rate (Westergren) $6 \mathrm{~mm}$. in first hour (normal 3 to 5 male, 4 to 7 female); Haemoglobin 14.4 g. -97 per cent.; Wbc. 7,000 per cmm.; Differential: polymorphs 78 per cent., lymphocytes 16 per cent., monocytes 6 per cent., eosinophils nil; red cells and platelets appear normal.

This examination was repeated on several occasions and the only different reading was that obtained on June 17, when the report was as follows:

Erythrocyte sedimentation rate (Westergren) $50 \mathrm{~mm}$. in first hour; Haemoglobin $14.9 \mathrm{~g}$. -101 per cent.; Wbc. 11,000 per c.mm.; Differential: Polymorphs 72 per cent., lymphocytes 17 per cent., monocytes 9 per cent., eosinophils 2 per cent.; red cells and platelets appear normal.

Numerous blood films were taken (at various hours of the day and night) while the patient remained in hospital, but at no time was a micro-filaria noted, despite very careful microscopic examination.

Stool examinations on two occasions were reported as: "No parasitic cysts, ova, or larvae detected".

An $x$ ray of the chest and skull showed no abnormality.

Operation.-The diagnosis having been confirmed, the patient was transferred to the theatre for removal of the parasite on June 6,1960. The anaesthetic was an intravenous 40 
injection of a mixture of promethazine hydrochloride, chlorpromazine, and pethedine hydrochloride in association with a retrobulbar injection of 2 per cent. Zylocaine. A left seventh nerve facial block was also given and lid sutures were used.

Following a suggestion arising from a past case report, a thin piece of black linen was cut so as to lie round the globe a few millimeters from the limbus and extending into the fornices, in the hope that when the white filaria was expelled from the eye it would lie in contrast upon a black background.

A keratome incision was made $1 \mathrm{~mm}$. beyond the limbus extending from 3.30 to 4.30 o'clock, in the hope that the filaria would shortly come to lie temporarily upon the iris in this area as it had frequently done during the period of observation. It was then intended to depress the posterior lip of the wound quickly, and expel the filaria within a gush of aqueous. Unfortunately, this was not to be, for the filaria within seconds of the completion of the section attached itself firmly by one end to the iris at about 9.30 o'clock -midway between the limbus and the pupil margin. After several minutes observation it appeared that it was the intention of the filaria to penetrate the iris tissue. An attempt to stroke the filaria free with the aid of an iris repositor gently applied to the outer surface of the cornea failed.

It was then decided to attempt to disengage the filaria by irrigating the anterior chamber with saline. This was preceded by a slight enlargement of the section with scissors in both the upward and downward directions. After several irrigations the iris prolapsed once, but the filaria remained in situ. The iris was replaced and a further irrigation attempted. Simultaneously the iris prolapsed for a second time and the filaria became dislodged and again freely mobile within the anterior chamber, where it remained until the iris was replaced. After several further minutes of activity it suddenly disappeared completely between the anterior lens capsule and the posterior surface of the iris, though fortunately within an area covered by the section.

In utter frustration, a complete iridectomy was undertaken, and when all was thought to be lost a fine wriggling movement was seen and the filaria was again noticed with one end adherent to the ciliary body region, as if it had commenced to burrow its way once more into uveal tisue.

After a further careful irrigation, the proximal free end of the filaria was washed forward, and its tip appeared just within the lips of the section long enough for it to be caught and extracted with a pair of iridectomy forceps. The section was enclosed with several 6.0 black silk sutures and the anterior chamber reformed by the injection of air.

Post-operative Course.-At the first dressing (24 hours after operation) the cornea was bright and clear, the anterior chamber was fully reformed, no hyphaema was present, and the lens was clear. There was no ocular discomfort.

These findings remained so until the morning of the sixth post-operative day, when the patient was awakened in the early hours by considerable pain in the affected eye. Examination now showed a complete hyphaema, the iris and the lens being totally obscured. This was followed for several days by increasing chemosis, oedema of both lids, and forward proptosis of the eye, associated with some limitation of ocular movements in all directions. The vessels of the conjunctiva became tortuous and dilated.

As a further orbital infestation was considered possible, the patient was again seen by a physician and under his guidance chloroquine was administered. Under this medication the proptosis, the oedema of the lids, and the chemosis partially resolved.

The hyphaema was being constantly reformed, despite oral adrenoxyl and systemic vitamin $\mathrm{K}$, until the 16 post-operative day when the blood absorbed rather quickly and it was noticed that the lens was now completely dislocated forward and was not only in contact with the posterior surface of the cornea but appeared completely opaque.

Examination under a slit lamp showed the iris vessels to be markedly dilated and new vessel formation appeared to be growing over the anterior lens capsule, especially in the 
area adjacent to the section which appeared fully healed with no leak. The cornea gradually became hazy with numerous folds in Descemet's membrane. No fundal detail or red reflex was present.

On the 26 th post-operative day the eye was completely hypotonic $(10 \mathrm{~mm}$. Hg. Schiötz), red, irritable, and very painful. As there was now no perception of light, the eye was enucleated.

The latter procedure provided its own problems, for the orbit was filled with large dilated and very tortuous vessels which bled profusely.

Pathological Report on the Enucleated Eye.-The eye was forwarded to Dr. C. H. Greer, of the Victorian Eye and Ear Hospital, Melbourne, who gave the following report:

Macroscopic Appearances: Left Eye.-There is scleral puckering posteriorly on the temporal side, the cornea is cloudy, and the pupil appears to be drawn towards the temporal side. Horizontal section above the disc is shown in Fig. 5. The rather thick retina is in place. The degenerate blood-stained vitreous has retracted centrifugally to form a thin layer over the retina and a large clot on the back of the lens, leaving the central vitreous cavity full of green watery fluid.

Fig. 5.-Section of globe.

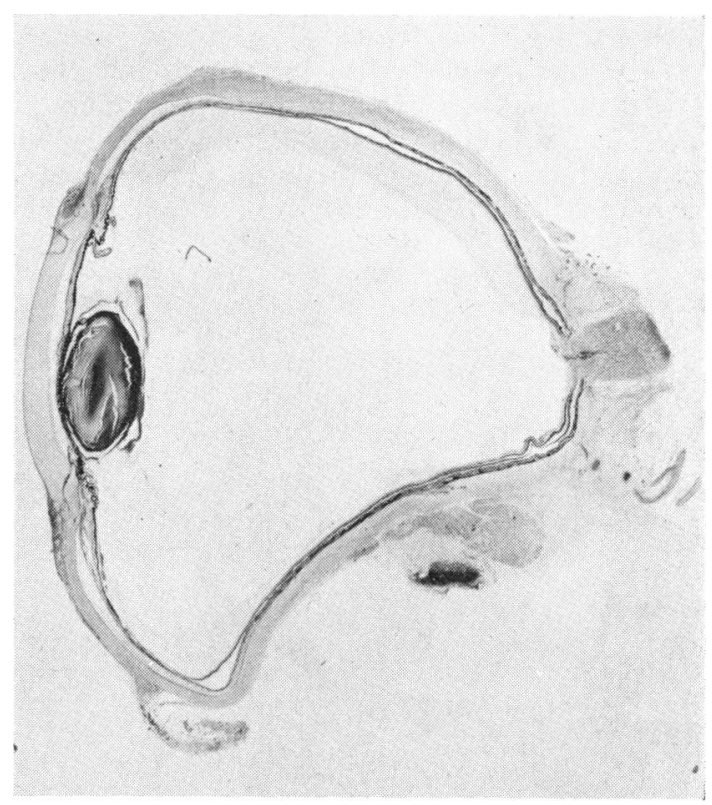

Sections.-These show the healed scar of an oblique penetrating wound near the limbus on the temporal side from the back of which scar tissue passes into the posterior chamber and also into the anterior chamber, where it replaces the temporal iris, fuses the large lens to the posterior corneal surface, and becomes adherent to the pupillary margin of the lower iris. The anterior substantia propria is infiltrated with chronic inflammatory cells, the remaining anterior chamber contains blood, and the lower filtration angle is partially closed by adhesions, in which new vessels are seen. The lens shows advanced degradation of its substance into small globules. Organizing haemorrhage is present in the iris, and haemorrhage and focal inflammatory infiltration in the ciliary body. Extensive serous effusion has occurred in the anterior suprachoroidal space above and below and there is 
mild focal choroiditis. The oedematous retina exhibits mild perivasculitis and there are many blood-ingesting macrophages in the vitreous layer on its surface. The central retinal vessels are patent. There is no eosinophilia and no evidence of any worm in multiple sections stained by haemotoxylin and eosin, periodic acid-Schiff, and Masson's techniques.

Comments. - On the basis of these findings, I would suggest that this eye was preoperatively in a state of metabolic decline and that the vitreous was already degenerate. Operation caused a collapse of vitreous structure, extensive choroidal detachment, and traumatic hypotony sufficiently severe to induce haemorrhage from granulation tissue at the operation site. It is not difficult to imagine that such events would cause reduced aqueous flow, and lens degeneration would follow rapidly, but may have been previously incipient. Loss of light perception would probably be due to the bloody vitreous deposits on the retina and behind the lens, degeneration and swelling of the lens, and scarring in the anterior segment.

Morphology of the Filaria.-After removal, the parasite was forwarded to Dr. T. Clive Backhouse of the School of Public Health and Tropical Medicine, the University, Sydney, to whom I am indebted for the following detailed report of the specimen:

Description of the Worm.-The specimen had been fixed in a coiled attitude and was received already cleared in lactophenol. Preliminary examinations and photographs were made with the worm in the same medium after which it was mounted in glycerin jelly. During manipulations, the attitude of the specimen was somewhat altered and the optical appearance of some internal structures changed. The present description and measurements refer to the specimen as seen in the original attitude as shown in Figs 6 and 7.

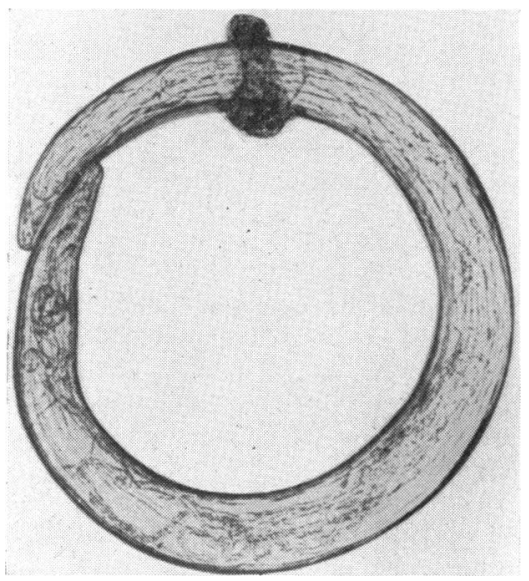

FIG. 6.-Low-power photomicrograph, showing general appearance of specimen with collarette of ocular tissue attached. $\times 20$.

FIG. 7.-High-power view of anterior and posterior ends of worm. $\times 160$. 
At the time of writing the specimen is in the care of Professor J. J. C. Buckley of the London School of Hygiene and Tropical Medicine, who is kindly carrying out some further studies, after the completion of which it will be lodged in the collection of the School of Public Health and Tropical Medicine, Sydney.

The worm was seen to be a small female nematode, measuring approximately 7.05 $\mathrm{mm}$. in length and having a maximum width of $0.35 \mathrm{~mm}$. The vulval orifice is $0.4 \mathrm{~mm}$. from the anterior end and leads to a coiled vaginal passage followed by a bifurcated uterus, which together with the ovarian tubes and ovaries forms a complicated mass of coiled structures occupying most of the body cavity of the worm. Fig. 8 shows a simplified representation with indications of the principle organs that could be identified.

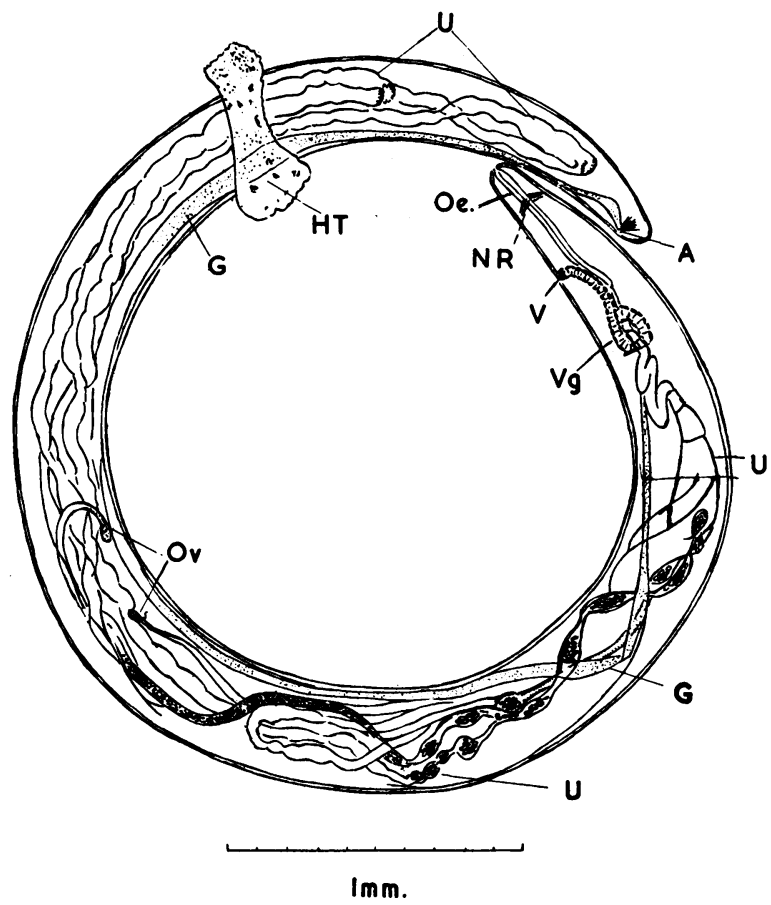

FIG. 8.-Drawing of worm (in part camera lucida). Some unidentified structures have been omitted for the sake of clarity.

Oe: oesophagus, N.R: nerve ring, V: vulva, Vg: vagina, U: uterus, G : gut, OV: commencement of ovaries, HT: collarette of host tissue, A: anus.

It has not been possible to determine the species or even the genus of this nematode. The supposition that it might be an immature female Wuchereria bancrofti receives no support from morphological study, and Professor Buckley has expressed the opinion that it belongs to one of the numerous species of filaria found in native animals, that has been fortuitously transferred to man.

Further and more detailed studies of the nematode are to be published in the Journal of Helminthology.

\section{Discussion}

This case history has been recorded, not only for its general ophthalmic interest, but because of the rarity of ocular filariasis and also, because it 
would appear to be the first time an intra-ocular filaria has been seen in Australia.

Fortunately, the specimen was collected without damage and could be subjected to minute morphological examination as reported by Dr. T. Clive Backhouse. At first, on clinical grounds, it was considered to be an immature form of Filaria bancrofti, but the detailed report on its characteristics makes this unlikely, although the specimen has not yet been definitely identified.

Cases of filariasis have been recorded in areas that are non-endemic, some years after the patient's return from an infested area. In this case the interval between the end of the patient's army service in the Middle East and the onset of the first clinical manifestations was some 17 years. It is unlikely that the parasite lay dormant during this long period, and it is more probable that the infestation originated in the North-eastern area of New South Wales shortly before the onset of symptoms.

Apart from the finding of an intra-ocular filaria, no other clinical sign indicated that filariasis of any kind was present. There was at no time a rise of temperature or pulse rate and no eosinophilia or micro-filaria were found on repeated blood examinations (day and night). This raises the question whether this type of filarial infestation may exist in tropical and subtropical areas of Australia in an asymptomatic form, for it is most unlikely that the only human infestation is one with an intra-ocular complication; in cases of filariasis the appearance of an intra-ocular filaria is purely a matter of chance and then only of a very remote one.

The parasite may have entered the eye in one of two ways:

(1) As a larval form from an invertebrate host (e.g. a mosquito or another biting insect).

On becoming larger it began to burrow through the ocular tissues in an attempt to get free and enter the lymphatic system, where the adults and maturing forms are usually found;

(2) As an immature or adult nematode.

It is unlikely that the parasite entered the eye as a larval form and there grew to some $7 \mathrm{~mm}$. in length without inducing earlier symptoms and signs that were completely absent in this patient.

The acute ocular onset suggests that the parasite entered the globe as an immature or adult form by direct burrowing through its outer coats or via a venous channel in the absence of ocular lymphatic vessels. In either case the nematode probably first invaded the ciliary body and then entered the anterior chamber by way of the iris tissues. This is in contradistinction to Onchocerca volvulus, which invariably enters the globe as a micro-filaria and never as an adult worm.

The ability of this particular filaria to burrow its way through tissues was well demonstrated, both at the time of the original consultation, when it had 
perforated the iris in three places, and later during the operation for its removal, when it was seen to attempt to enter not only the iris but also the ciliary body.

\section{Summary}

A case of an unidentified intra-ocular filaria is reported. The case presented as an iridocyclitis and details of the operative removal of the filaria and the attendant difficulties are recorded. The eye progressed most satisfactorily for 6 days following the operation, but eventually required enucleation (22nd day) because of dislocation of the lens, hypotonia, and loss of perception of light.

The pathological findings of the eye and the morphology of the filaria are reported in detail.

It is suggested that further asymptomatic and unrecognized cases of this nematode may exist in the tropical and sub-tropical areas of Australia.

To Dr. T. Boyd Law, who first examined this case, Dr. T. Clive Backhouse, for his detailed report of the filarial specimen, and Dr. C. Greer, for the pathological details, I acknowledge my sincere thanks and gratitude.

Appreciation is also expressed to Prof. J. J. C. Buckley of London, to whom the specimen was forwarded for further opinion.

\section{REFERENCE}

Duke-Elder, S. (1940). “Text-book of Ophthalmology”, vol. 3, pp. 3430-3450. Kimpton, London. 